\title{
Producto turístico patrimonial para el batey azucarero Jaronú, desde un enfoque multidisciplinario.
}

\author{
Heritage tourism product for the Jaronú sugar mill, from a \\ multidisciplinary approach.
}

\author{
Dr. C. Gerson Herrera Pupo. ${ }^{1}$, Dr. C. María Elena Betancourt García. ${ }^{2}$ \& MSc. Geiser \\ Perera Téllez ${ }^{3}$
}

\begin{abstract}
The province of Camaguiey is one of the most significant territories for Cuba's agroindustrial sugar heritage. Among its main exponents is the Jaronú sugar plantation declared in 2008 as a National Monument and which has historical, heritage and cultural singularities that merit its enhancement for tourist purposes as a complementary attraction to the prospective tourist development of the north of Camaguey. In this sense, the present work aims to show the multidisciplinary interrelation achieved at the Center for Multidisciplinary Tourism Studies (CEMTUR) of the University of Camaguiey for the design of a heritage tourism product linked to the Jaronú sugar mill, as a faithful reflection of the culture - tourism relationship. The aforementioned interrelation is demonstrated starting from multidisciplinary conceptualization works on industrial heritage, the establishment of historical, social and cultural conditions for the development and consolidation of the sugar industry in the region, passing through studies of the potential and hierarchization of its attractions, the concretion of conceptual ideas for the design and operation of a visitor center in the referred batey, until reaching the conceptual design of a tourist product linked to the site.
\end{abstract}

\footnotetext{
${ }^{1}$ Profesor Titular, Centro de Estudios Multidisciplinarios del Turismo (CEMTUR) de la Universidad de Camagüey "Ignacio Agramonte Loynaz" - Cuba, Circunvalación norte KM 5 1⁄2 Camagüey - Cuba CP74650, Teléfono: 32-266307, gerson.herrera@reduc.edu.cu

2 Profesora Titular, Directora del Centro de Estudios Multidisciplinarios del Turismo (CEMTUR) de la Universidad de Camagüey "Ignacio Agramonte y Loynaz" - Cuba, Circunvalación norte KM 5 1⁄2 Camagüey - Cuba CP74650, Teléfono: 32-266307, elena.betancourt@ reduc.edu.cu

${ }^{3}$ Master en Gestión Turística, Profesor Auxiliar, Centro de Estudios Multidisciplinarios del Turismo (CEMTUR) de la Universidad de Camagüey "Ignacio Agramonte y Loynaz" - Cuba, Circunvalación norte KM 5 1⁄2 Camagüey - Cuba CP74650, Teléfono: 32-266307, geiser.perera@ reduc.edu.cu
} 
Key Words: Sugar Heritage; Put In Value; Complementary Attractive; Patrimonial Tourist Product.

\section{Resumen}

La provincia de Camagüey, es uno de los territorios de mayor significación para el patrimonio agroindustrial azucarero de Cuba. Dentro de sus principales exponentes se encuentra el batey azucarero Jaronú declarado en el 2008 Monumento Nacional y que posee singularidades históricas, patrimoniales y culturales que ameritan su puesta en valor con fines turísticos como atractivo complementario al desarrollo turístico perspectivo de la zona norte camagüeyana. En este sentido, el presente trabajo tiene como objetivo mostrar la interrelación multidisciplinaria lograda en el Centro de Estudios Multidisciplinarios del Turismo (CEMTUR) de la Universidad de Camagüey para el diseño de un producto turístico patrimonial vinculado al batey azucarero Jaronú, como fiel reflejo de la relación cultura - turismo. Se demuestra la referida interrelación partiendo de trabajos multidisciplinarios de conceptualización sobre patrimonio industrial, el establecimiento de las condicionantes históricas, sociales y culturales para el desarrollo y consolidación de la industria azucarera en la región, pasando por los estudios del potencial y la jerarquización de sus atractivos, la concreción de ideas conceptuales para el diseño y funcionamiento de un centro de visitantes en el referido batey, hasta llegar al diseño conceptual de un producto turístico vinculado al sitio.

Palabras Clave: Patrimonio Azucarero; Puesta En Valor; Atractivo Complementario; Producto Turístico Patrimonial.

\section{Introducción}

En los últimos años el turismo ha ido adquiriendo una notoria importancia que se manifiesta en el aumento del número de personas que se desplazan con fines turísticos de un lugar a otro, y en la búsqueda permanente de nuevas modalidades turísticas y en expansión y de la formulación de nuevos productos.

Ello presupone la creación de nuevos escenarios turísticos de futuro, donde se evite el deterioro que han sufrido aquellos productos tradicionales, vinculados a un uso indiscriminado de los recursos y a la ausencia de objetivos a largo plazo, por lo que el turismo se ha convertido en un elemento clave para la mejora de la calidad de espacios degradados y garantizar el mantenimiento y la preservación del patrimonio cultural.

El territorio camagüeyano, como una de las regiones más significativas de la agroindustria azucarera tanto en Cuba como en el escenario internacional, posee características peculiares que lo diferencian con respecto al resto del país, debido fundamentalmente a sus condicionantes, su formación y evolución.

Lo anteriormente planteado justifica la necesidad de realizar estudios que se complementen a las singularidades y potencialidades patrimoniales de la agroindustria azucarera en Camagüey. Específicamente estas necesidades han tenido como objeto de acción el batey azucarero Jaronú, ubicado en el centro de influencia de la actual zona 
turística en desarrollo de la cayería norte de la provincia donde se prevé un desarrollo turístico acelerado a corto plazo y donde el estado cubano ha apostado con el máximo de recursos disponibles.

En este sentido, se considera la necesidad inminente de gestionar turísticamente el batey azucarero Jaronú a partir de: la carencia de productos turísticos autóctonos que revelan los valores más significativos del referido exponente del patrimonio industrial azucarero; la carencia de herramientas metodológicas que desde la perspectiva de la interpretación permitan gestionar adecuadamente este patrimonio; la necesidad por parte del MINTUR a escala nacional de potenciar con fines turísticos este atractivo del potencial industrial azucarero y su conversión en producto turístico; la creación de instrumentos que contribuyan a consolidar un producto turístico y temático del azúcar (ruta del azúcar Jaronú - Esmeralda); la necesidad de consolidar los valores de este espacio patrimonial de forma que la comunidad y los visitantes se identifiquen con estos valores; y la deficiente estrategia de promoción del batey azucarero Jaronú como atractivo turístico capaz de generar desarrollo a escala local y territorial.

Es por ello que el presente trabajo tiene como objetivo mostrar la interrelación entre varias disciplinas lograda en el Centro de Estudios Multidisciplinarios del Turismo (CEMTUR) de la Universidad de Camagüey para el diseño de un producto turístico patrimonial vinculado al batey azucarero Jaronú. Todo ello partiendo de la necesaria conceptualización sobre patrimonio industrial y patrimonio agroindustrial azucarero, así como el establecimiento de las condicionantes para su desarrollo y consolidación, pasando por los estudios del potencial y la jerarquización de sus atractivos, la concreción de ideas conceptuales para el diseño y funcionamiento de un centro de visitantes en el referido batey, hasta llegar al diseño conceptual de un producto turístico vinculado al sitio.

\section{Desarrollo}

La concreción de una primera etapa de lo aquí presentado ha permitido formar parte del proceso de identificación, reconocimiento y caracterización de los valores patrimoniales que posee el referido batey azucarero y con ello ser parte justificativa para su declaratoria como Monumento Nacional por la Comisión Nacional de Monumentos en el 2008. Esta declaratoria ha propiciado estatus de protección a los valores patrimoniales singulares dado que es fiel reflejo de una etapa de la vida productiva y económica de la nación y mostrar aún de forma viva su desarrollo.

A su vez, los estudios realizados en torno a la identificación de su potencial turístico y la conceptualización de posibles productos turísticos vinculados al batey azucarero ya forman parte de la cartera de futuros productos turísticos complementarios de la zona norte Camagüeyana, una de las ocho priorizadas a nivel de país.

Se presentan cronológicamente y de acuerdo a un esquema metodológico investigativo y exhaustivo cada etapa de desarrollo del trabajo que abarca dos décadas de investigaciones 
continúas desarrolladas por varios equipos de profesores y estudiantes de varias especialidades (arquitectura, estudios socioculturales, turismo, etc.) todos liderados por los referidos autores del CEMTUR.

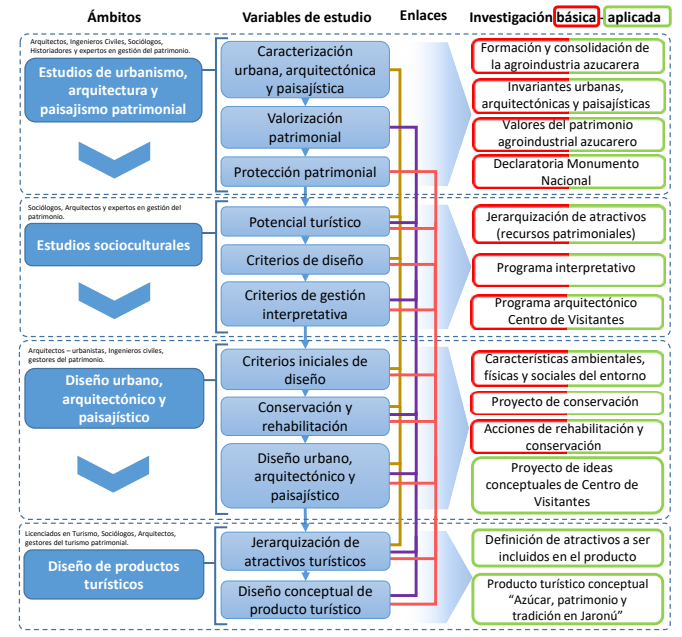

Figura 1: Esquema metodológico de integración multidisciplinaria del proceso de creación de productos turísticos para el batey azucarero Jaronú.

Fuente: Elaboración propia

Como se muestra en la figura 1, el referido esquema metodológico que evidencia la integración multidisciplinaria del proceso de creación de un producto turístico que ha sentado las bases para la declaratoria de Monumento Nacional y a su vez deja establecido criterios a seguir para la implementación de futuros productos turísticos en el batey azucarero Jaronú y su área de influencia (paisaje asociado).

Se ha de tener en cuenta que este ha sido un proceso metodológico basado en estudios histórico lógicos y de observación analítica con criterios sistémicos y de análisis y síntesis de toda la información obtenida y acumulada a lo largo de los años de investigación y que comenzaron con el proceso de reordenamiento de la agroindustria azucarera en el año 2002 (Tarea de estado Álvaro Reynoso).

A continuación se desarrolla resumidamente la exposición de cada uno de los ámbitos donde se aprecia la integración multidisciplinaria que propició el crear investigaciones básicas y aplicadas de forma concatenadas y que han facilitado el contar en la actualidad con una detallada fuente documental para enfrentar futuros diseños de productos turísticos para el batey azucarero Jaronú u otro sitio patrimonial similar.

El urbanismo, la arquitectura y el paisaje del batey azucarero jaronú como bien patrimonial.

\section{Formación y consolidación de la agroindustria azucarera:}

La agroindustria azucarera cubana es reconocida por su valiosa historia y por ser fuente de identidad multicultural. A decir de Miguel Barnet (2005, p. 11) "Fue el origen de todo nuestro ser, la casa donde se gestó nuestra personalidad”. Su formación y consolidación se mantuvo relacionada con disímiles condicionantes que permitieron establecer un 
escalonado y diferenciado sistema agroindustrial azucarero cuyas características se corresponden directamente con la historia de la nación.

La agroindustria azucarera cubana, durante poco más de cuatro siglos, experimentó cambios debido a diversas condicionantes socio-políticas, económico-tecnológicas, ambientales y culturales, que propiciaron su expansión y desarrollo. Se sucedieron desde tiempos de la Colonia hasta pocos años antes de 1959 tres grandes acontecimientos que marcaron el auge económico y el desarrollo constructivo agroazucarero en el país: El embargo comercial de Estados Unidos (1803-1808), las últimas Guerras de independencia de la metrópoli española (1886.1899), y la crisis mundial (1928-1933).

Posterior a 1959 la agroindustria azucarera experimentó cambios sustanciales en lo concerniente al modo de producción y a su relación social. La socialización de la producción, y la introducción de nuevas tecnologías facilitaron el incremento productivo, así como la construcción de nuevos centrales azucareros. Sin embargo, los precios impuestos internacionalmente, la falta de mantenimiento sostenido y de adquisición de insumos, entre otras condicionantes, llevaron a que en el año 2002 se reestructurara nuevamente el sistema agroazucarero en todo el país.

Ante la pérdida consecutiva de los principales atributos materiales e inmateriales vinculados a la agroindustria azucarera cubana, el patrimonio agroindustrial azucarero necesita de acciones de identificación y reconocimiento de sus valores patrimoniales con el fin de concatenar criterios para su protección y puesta en valor.

En la actualidad, la mayoría de los Centrales Azucareros, y sus respectivos componentes vinculados a la producción, ya sea la industria, el ferrocarril, las plantaciones, o el batey, aún persisten físicamente. Estos componentes se han visto amenazados por determinados procesos o sucesos acaecidos de forma directa e indirecta, lo que ha conllevado a la inminente pérdida de su autenticidad y de su integridad. El componente que mayores impactos ha sufrido desde el punto de vista socio - económico es precisamente el batey azucarero, que, anexo a la industria, tiene la función de brindar alojamiento a los administrativos, obreros y sus familias y ha llegado hasta hoy con un marcado deterioro físico.

La provincia de Camagüey, reconocida durante varios siglos como productora exclusiva de ganado, poseía desde el siglo XVII pequeñas manufacturas azucareras productoras de mascabado y raspaduras. Diversos sucesos políticos y económicos hacen que la región dé un giro en su tipo productivo, al pasar, de mayor productor ganadero nacional, a máximo productor azucarero a nivel internacional. El cambio fue favorecido por la construcción, en la provincia, de grandes centrales azucareros formados por una red de componentes vinculados directa o indirectamente con el proceso productivo azucarero. Con la construcción de las vías férreas, y debido a condicionantes externas en Camagüey, se fomentaron desde finales del siglo XIX y durante los 50 años posteriores cuatro concentraciones agroindustriales azucareras (Herrera, 2008). 


\section{El batey azucarero jaronú. Monumento nacional:}

El conjunto agroindustrial azucarero Jaronú (construido en 1921) presenta la mayor calidad visual, integridad y autenticidad de sus elementos componentes. La integridad paisajística se refuerza por la atipicidad de su contraste cromático favorecido por el rojo de sus suelos y los campos de cultivo, así como por la existencia de hallazgos arqueológicos en sus límites, y por ser sitio donde ocurrieran hechos históricos de relevancia internacional. Posee el asentamiento agroindustrial azucarero de mayor tamaño, con el central azucarero de mayor capacidad, y con el batey azucarero de mayor integridad, autenticidad y atipicidad de toda la muestra, ambos construidos con capital norteamericano (Herrera, 2008, p.158).

$\mathrm{Su}$ batey azucarero es el mejor estructurado formal y funcionalmente, con un detallado sistema de zonas funcionales. La estructura vial es la de mejor estado de conservación y homogeneidad, y, de conjunto con el parque de mejor diseño de la muestra, se complementa la alta calidad paisajística del batey. La homogeneidad de los códigos arquitectónicos atípicos en sus edificaciones, y de las técnicas constructivas novedosas con respecto a su época y a sus similares, así como su buen estado de conservación, hacen que sea reconocido internacionalmente (Herrera, 2008, p.158)

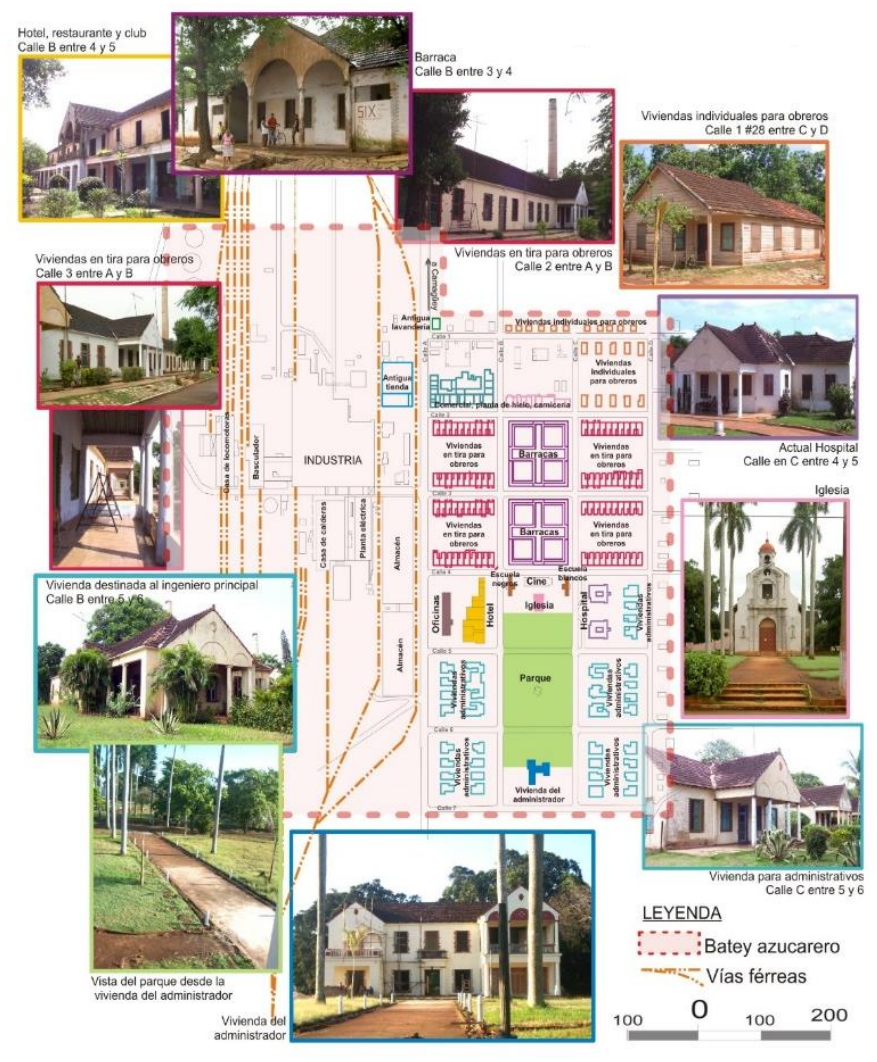

Figura 3: Estructura urbana y principales partes y elementos componentes del batey azucarero Jaronú.

Fuente: Herrera, 2008, p. 90 
La aplicación del proceso de valoración a nivel provincial arrojó que, de los seis conjuntos agroindustriales azucareros seleccionados, la mayoría posee mucho valor; mientras que Jaronú es el único que posee valor patrimonial excepcional. La investigación de Herrera (2008) permitió identificar a su vez Junto al Jaronú a otros cinco conjuntos agroindustriales azucareros como una concentración que, evaluada de acuerdo con los procedimientos establecidos por la UNESCO, permite ser identificada como un bien patrimonial del tipo paisaje cultural evolutivo vivo, por conservar vestigios de la función social e industrial, y tener características urbanas, arquitectónicas, paisajísticas e inmateriales íntegras, auténticas y atípicas.

\section{Criterios para el diseño del centro de visitantes del patrimonio agroindustrial azucarero en el batey azucarero Jaronú.}

La interpretación del patrimonio como disciplina y herramienta de gestión permite poner en práctica diversos proyectos, concretados en la implementación de medios y equipamientos interpretativos para lograr objetivos vinculados a la preservación y difusión del legado patrimonial. Su surgimiento está relacionado con la necesidad de comunicar y hacer consciente al visitante o persona que visita un bien patrimonial sobre la necesidad de conservar y difundir sus más significativos valores, atravesado por la práctica de actividades de uso público que logren hacer compatible el uso y disfrute del bien visitado (Luís, 2012, p. 7).

Uno de los equipamientos de relevante importancia para alcanzar la meta interpretativa lo constituyen los Centros de Visitantes, equipamientos acondicionados especialmente para hacerle llegar al visitante de forma activa y creativa todo el conocimiento y los valores patrimoniales más significativos del lugar que visita (Luís, 2012, p. 7).

En Cuba se ha puesto en práctica esta modalidad, con la implementación de Centros de Visitantes en varias ciudades importantes del país, apoyado en las ventajas que estos brindan como equipamientos al servicio de la promoción e interpretación del patrimonio.

En el marco del actual modelo de gestión económica que impulsa el estado cubano, la promoción del patrimonio como vía para lograr captar ingresos por concepto de turismo constituye un factor clave de éxito; máxime si se tiene en cuenta que la industria del turismo está llamada a ser el sector más dinámico de nuestra economía; que ha venido experimentando en los últimos años un crecimiento vertiginoso, con resultados sustanciales en el plano económico y sociocultural. En tal sentido los Centros de Visitantes devienen en estrategia fundamental, pues constituye un equipamiento de incuestionable valor concebido para la interpretación y promoción del patrimonio.

\section{Pasos metodológicos para la definición de los criterios de diseño:}

Dentro de este ámbito de la investigación se siguieron tres pasos metodológicos para la definición de los criterios de diseño del Centro de Visitantes del batey azucarero Jaronú (Luís, 2012, p.37). 
Paso 1: Definir potencialidades para la interpretación del patrimonio agroindustrial azucarero en Jaronú.

Paso 2: Indicadores para la definición de criterios para el diseño del Centro de Visitantes.

Paso 3: Criterios para el diseño del Centro de Visitantes.

Los pasos anteriormente señalados describen los momentos que se han tenido presentes en la investigación para llevar a cabo la definición de los criterios de diseño para el Centro de Visitantes.

El primer momento dedicado a la definición de las potencialidades patrimoniales a interpretar en el Centro de Visitantes, ya que previamente a la etapa del diseño de estos equipamientos se requiere conocer las potencialidades patrimoniales del lugar y aquellas que por sus altos valores merecen ser potenciadas por encima de otras.

En la misma se agruparon por repertorios o conjuntos aquellos recursos patrimoniales con atractivo turístico que se encuentran en el batey Jaronú. Estos son:

- Conjunto industrial: Comprende todos los edificios que intervienen en el proceso productivo azucarero, con excepción de los talleres ferroviarios.

- Conjunto ferroviario: este comprende los talleres ferroviarios, las vías férreas y la estación de viajeros.

- Batey azucarero: comprende todo el repertorio habitacional y los edificios singulares de carácter social.

En un segundo momento se definieron los indicadores pertinentes para el diseño del Centro de Visitantes teniendo en cuenta los criterios planteados por los expertos a través de la aplicación del instrumento para la recogida de información, en este caso, la entrevista estructurada (Luís, 2012, p.44).

En lo referido al aspecto físico los expertos consideraron que el Centro de Visitantes debe estar marcado por la originalidad de su diseño, la mayoría coincidió en que este debe respetar los cánones que rigen la arquitectura local y por tanto estar integrados al entorno, de forma tal que los habitantes de la comunidad se logren identificar con el inmueble, así como con las funciones a las cuales está dirigido.

Los expertos coinciden en que el diseño del Centro de Visitantes debe tener una estructura de tipología industrial, que semeje la del antiguo central azucarero, que es el eje temático del conjunto, alrededor del cual se concentra toda la trama histórica y arquitectónica y que atesora gran parte del cúmulo de valores patrimoniales industriales del lugar.

Se establecieron de forma general los siguientes indicadores pertinentes para el diseño del Centro de visitantes en el batey azucarero Jaronú:

- Diseño acorde con la arquitectura local.

- $\quad$ Evitar las construcciones sobredimensionadas o excesivamente vanguardistas. Debe de estar integrado con el medio, para ser capaz de promover los valores patrimoniales del lugar. 
- $\quad$ Procurar no copiar otros diseños. El equipamiento debe estar adaptado a las características y materiales propios de la localidad. La autenticidad del diseño facilitará la eficacia del equipamiento posibilitando su integración en el entorno.

- Adaptar completamente el edificio a personas con necesidades especiales.

- Tener en cuenta las medidas de seguridad propias de locales públicos, como salidas de emergencia, alarmas contra incendios, etc.

- Aprovechamiento detallado de las técnicas de interpretación patrimonial.

En el tercer y último momento se proponen los criterios para el diseño del Centro de Visitantes, con los elementos antes desarrollados y explicados para el caso del batey azucarero Jaronú. Esta información responde a los planteamientos realizados por los expertos, los cuales son considerados de gran interés para enriquecer el resultado de esta investigación y aportar elementos suficientes para justificar y su diseño.

El edificio que acogerá al centro de Visitantes deberá cumplir con los siguientes criterios de diseño (características arquitectónicas):

- $\quad$ Debe ser una construcción de tipo industrial, teniendo en cuenta que deberá estar ubicado cercano a la zona de la industria (central azucarero).

- La fachada estará en correspondencia con las características del entorno, teniendo presentes los elementos arquitectónicos que predominan en él, aunque contará con algunos motivos más contemporáneos, como cristales y el uso de estructuras compuestas (acero - hormigón).

- Se utilizará como material constructivo el mampuesto para los muros (cierre vertical) y la cubierta deberá ser preferiblemente de zinc galvanizado con un elevado puntal manteniendo la escala del conjunto.

- Para su construcción se aprovecharán como sistema portante las vigas y columnas existentes en el sitio.

- Se desarrollarán espacios funcionales acordes a las necesidades a cubrir, siendo las salas de medios interpretativos la que condicionen la estructura funcional.

\section{Programa arquitectónico general:}

Para el emplazamiento del Centro de Visitantes se seleccionó el espacio de lo que fuera parte de uno de los almacenes pertenecientes al central, teniendo en cuenta la amplitud del área que ocupa, de ahí que pueda brindar una capacidad de carga mucho mayor y la posición en que se ubica con respecto al Central (industria), a las vías férreas y al batey azucarero.

El Centro de Visitantes del batey azucarero Jaronú deberá poseer nueve espacios funcionales que se desarrollarán a partir de un espacio relacionador que es la recepción. Estos espacios se agrupan en tres zonas funcionales específicas: la zona funcional de servicio (Recepción, tienda y cafetería), la zona funcional de apoyo (Biblioteca, aula taller y sala de conferencia), y la zona funcional interpretativa (estación, sala Jaronú y sala de 
exhibiciones). En la figura 4 se observa el esquema de relación funcional de todos los espacios.

Como parte del sistema de actividades interpretativas propuestas que comprende medios interpretativos tradicionales (maquetas, poster, audiovisuales, piezas museísticas, etc.), se propone como medio interpretativo personal un recorrido temático en tren desde el batey azucarero hasta la estación de viajeros distante 2 kilómetros del referido Centro de Visitantes.

El recorrido se realizará en tren teniendo en cuenta la importancia que reviste sus valores patrimoniales y significación en la historia de la localidad. Este recorrido resulta de mucha importancia al incluir la presencia de un guía o intérprete que puede otorgar mayor riqueza a la interpretación, ya que es la comunicación verbal la forma más directa de transmitir el mensaje al público. De su destreza y habilidad para captar la atención de los visitantes dependerá en gran medida el éxito del paseo.

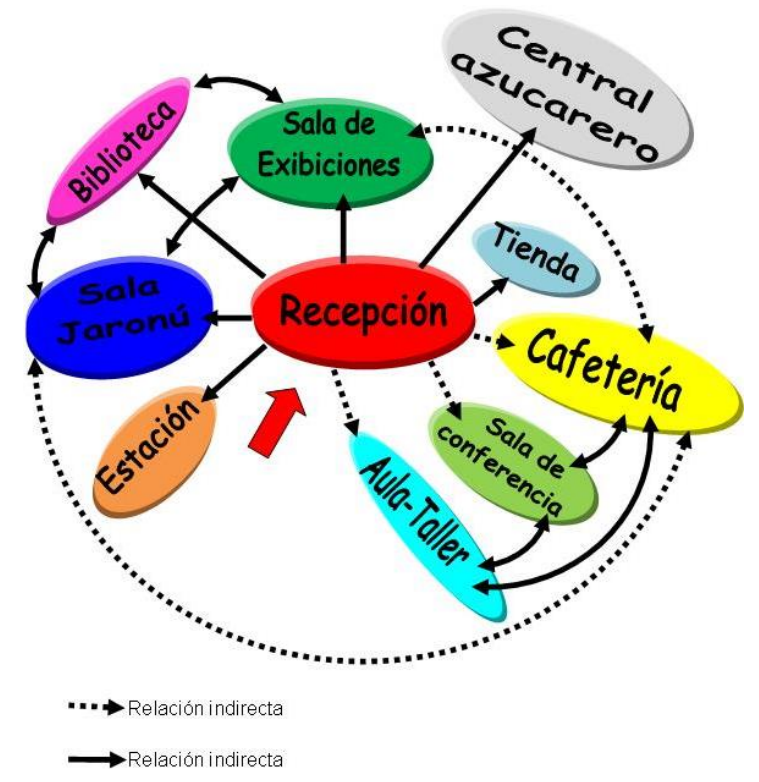

Figura 4: Esquema de relaciones

funcionales del Centro de Visitantes del batey azucarero Jaronú.

Fuente: Luís, 2012, p. 51

Este tren a su vez funcionará como medio de transporte a los pobladores locales con la finalidad de dirigirse hasta la estación de viajeros, logrando así dar respuesta a uno de los principales problemas sociales de los pobladores y a su vez permitirá la interacción de los visitantes con la población local.

El tren deberá estar acondicionado al efecto y se trasladará por el ramal que conecta al batey azucarero con la estación de viajeros, el regreso se realizará por la misma ruta, permitiendo apreciar el procedimiento llevado a cabo en el patio ferroviario, así como los valores patrimoniales y paisajísticos del entorno. 
Tema a desarrollar en el recorrido: "Acercamiento al patrimonio ferroviario vinculado a la agroindustria azucarera del conjunto agroindustrial Jaronú."

Título: "Ferrocarril azucarero Jaronú".

Objetivos:

1- De conocimiento: que el visitante sea capaz de reconocer los valores patrimoniales del conjunto agroindustrial azucarero Jaronú y su ferrocarril como los exponentes representativos del patrimonio agroazucarero cubano.

2- Emocional: que el visitante sienta y viva la importancia histórica y patrimonial que encierra el batey azucarero Jaronú.

3- De actitud/comportamiento: que el visitante adopte un comportamiento que no afecte negativamente el entorno y que contribuya a su conservación.

\section{Proyecto arquitectónico de centro de visitantes del patrimonio agroindustrial azucarero en el batey azucarero jaronú.}

Las integraciones de partes nuevas en edificios existentes, así como de nuevos edificios en contextos de valor han constituido en cada periodo de la arquitectura, una respuesta de solución urbano-arquitectónica al deterioro lógico y la necesidad de las transformaciones que se van produciendo con el paso del tiempo.

Para integrar lo nuevo con lo viejo se hace necesario hacer una integración que este motivada por criterios composicionales, lo que no significa realizar una copia de la arquitectura pasada, sino un estudio de sus características orgánicas de aquellos criterios que han sido retomados de una etapa u otra y que en síntesis han brindado la continuidad necesaria en la evolución histórica de la vieja ciudad (Brolin, 1984).

Para la realización del proyecto arquitectónico (proyecto de ideas conceptuales) se procedió a diseñar un esquema (Ver figura 5) que muestra la vinculación de los espacios ya establecidos en el programa arquitectónico definido en el ámbito precedente (Criterios de diseño), los cuales estarán organizados en los dos niveles previstos y conectados por una escalera y una rampa para personas con limitaciones especiales.

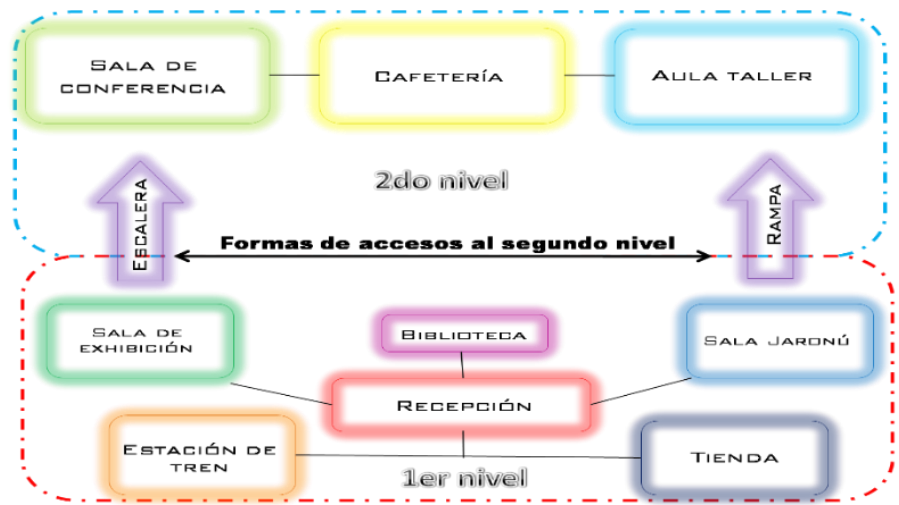

Figura 5: Vinculación de los espacios del centro de visitantes del batey azucarero Jaronú.

Fuente: Santisteban y Hernández, 2012, p.43 
Se definieron tres variantes de zonificación y relación de las zonas funcionales y cuatro variantes de estructuración volumétrica del centro de visitantes del batey azucarero Jaronú como parte del proceso lógico de diseño arquitectónico que plantea la aproximación sucesiva a la solución final. Se logró finalmente un edifico funcional y estéticamente integrado al entorno con elementos identitarios de la arquitectura propia de los edificios industriales (altos puntales, estructuras de acero y hormigón, cubiertas metálicas inclinadas) y motivos figurativos devenidos de la arquitectura doméstica predominante en el batey azucarero (cubiertas inclinadas, arcos, amplios vanos, pisos y mobiliario, lambrequines, cartelas, aleros de tornapunta, etc.) (Ver figura 6).
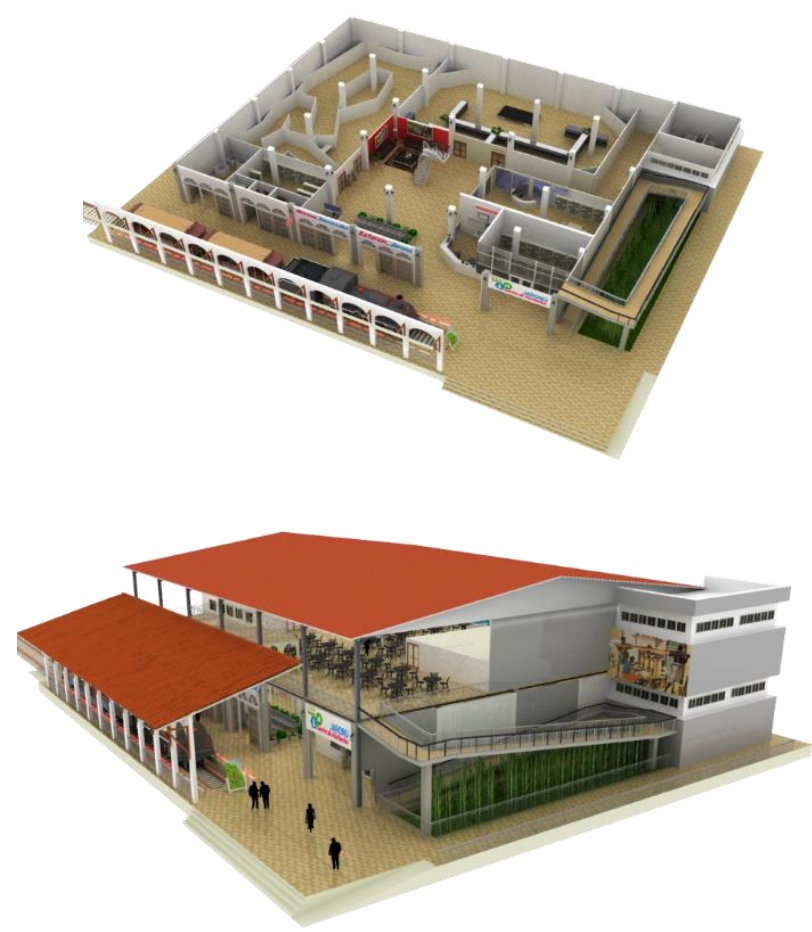

Figura 6: Planta arquitectónica y volumetría del Centro de Visitantes del batey azucarero Jaronú.

Fuente: Santisteban y Hernández, 2012, p.60 y 61

\section{Formatos promocionales del centro de visitantes del batey azucarero Jaronú:}

Como parte del proceso de diseño arquitectónico se añadieron a este ámbito la propuesta de tres elementos propios del diseño gráfico y documental que aportan identidad al proyecto arquitectónico del centro de visitantes y complementan el producto turístico y la puesta en valor de sus características patrimoniales.

En primer lugar se diseñó un mapa temático que agrupa las principales características y condiciones de accesibilidad y de relaciones del batey y sus valores patrimoniales. En segundo lugar se conformó un video promocional del centro de visitantes y su vínculo con el batey azucarero (ver figura 8). Y finalmente se diseñó una señalética afín (ver figura 9). 


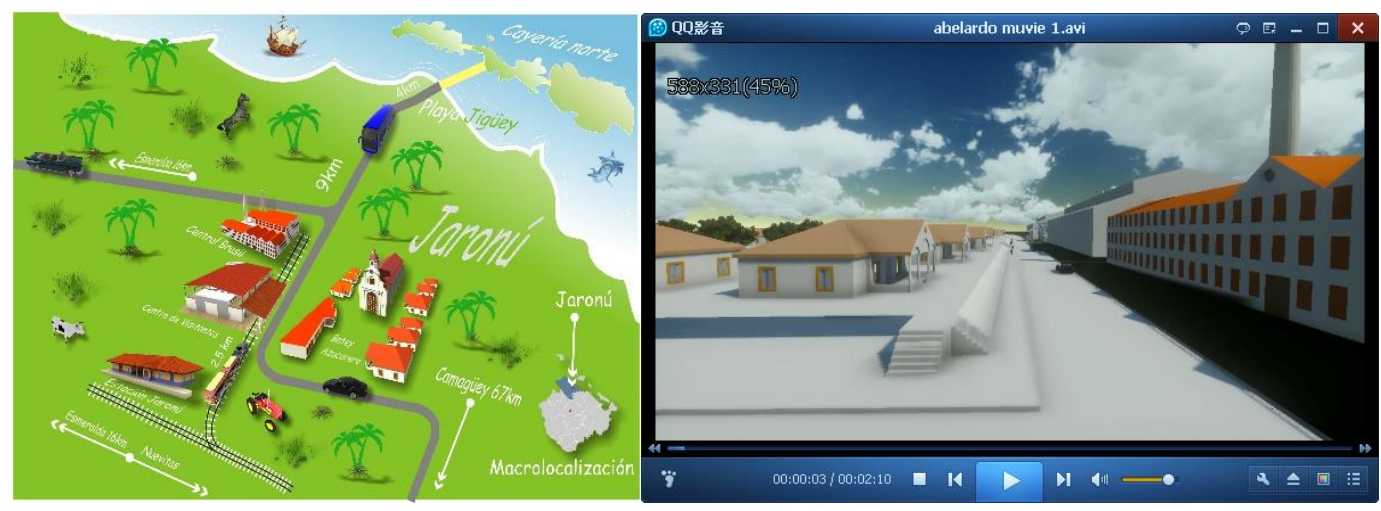

Figura 7: Mapa temático y video promocional del Centro de Visitantes del batey azucarero Jaronú.

Fuente: Santisteban y Hernández, 2012, p.73 y 74

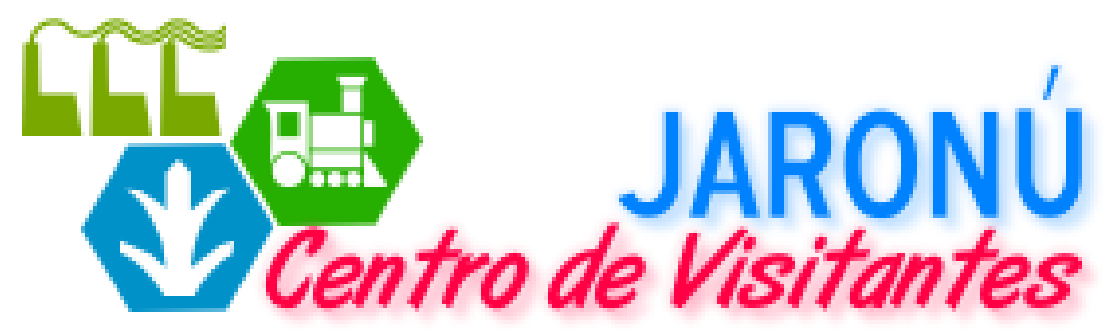

Figura 8: Señalética del Centro de Visitantes del batey azucarero Jaronú.

Fuente: Santisteban y Hernández, 2012, p.75

\section{Producto turístico conceptual "azúcar, patrimonio y tradición en Jaronú”.}

A partir de todo el respaldo investigativo y de proyección realizado por cerca de dos décadas teniendo como objeto de investigación al batey azucarero Jaronú y a sus características y valores patrimoniales, se han desarrollado diversos acercamientos a posibles productos turísticos de forma conceptual. Sin embargo, recién se acaba de concretar un producto turístico conceptual (Pimentel, 2019) que integra de forma consciente los diversos ámbitos expresados en el presente trabajo.

Para el desarrollo conceptual del producto “Azúcar, patrimonio y tradición en Jaronú" se asumió la "Metodología para el diseño de productos turísticos" del Centro de Estudios de Gerencia, Desarrollo Local y Turismo; GEDELTUR de la Facultad de Ciencias Económicas de la Universidad de Pinar del Río (Gómez, 2012) por poseer una estructura de diseño específica y sistemática acorde al presente trabajo, y a que describe de forma detallada el procedimiento para el desarrollo de un producto turístico y estar enmarcado 
en Cuba. Es necesario señalar que para el diseño del presente producto se desarrollaron solamente dos fases completas del procedimiento y una parte de la tercera fase, toda vez que se ha desarrollado en un primer momento como producto conceptual.

\section{Fase I: Proceso de Identificación de la Demanda}

En el caso de la presente investigación se trabajó con un estudio de campo basado en mercados futuros, con clientes que acceden mayoritariamente al producto como opcional complementaria obtenida desde uno de los hoteles de sol y playa del destino Cayo Cruz quien será el principal emisor. Se prevé que una vez concretada la etapa de diseño definitivo del producto se deberá realizar el análisis de las tendencias actuales de los principales mercados emisores, así como, su comportamiento, y motivaciones.

Se propone que el producto esté dirigido fundamentalmente a grupos de personas con un alto nivel cultural, que aprecian y respetan los bienes culturales, que generalmente sean viajeros frecuentes, educados y amigables en sus encuentros con personas de otras culturas. Se prevé además que se caractericen en particular por: ser personas adultas en plena madurez, o jóvenes con amplios conocimientos culturales entre 30 y 60 años, tener un nivel educativo y económico superior al turista medio, tener la posibilidad de desplazarse en épocas de temporada baja. Que muestren interés por desplazamientos geográficos por el interior, ser exigentes con los servicios que recibe, que decidan el producto con antelación en función de sus intereses culturales.

\section{Fase II. Diseño del Producto.}

Se identificaron los principales recursos (atractivos) tangibles e intangibles que posee el batey azucarero y que están en correspondencia con la definición de las características y valores patrimoniales que poseen estos recursos determinados en el primer ámbito del presente trabajo.

Se definieron como principales atractivos tangibles para el diseño conceptual del producto: La iglesia, la antigua casa del administrador, el parque, las escuelas, las cuarterías (barracas), el hotel, la estación de ferrocarril y el central. Todos fueron debidamente caracterizados de acuerdo a su potencial como atractivo patrimonial susceptible de convertirse en atractivo turístico (Pimentel, 2019, p. 41 a 47). Como atractivos tangibles se caracterizó la imagen formal y social del batey; los hechos históricos relevantes; en lo cultural la estructuración social y el acervo cultural del asentamiento; la originalidad del batey por su estructura; el reconocimiento exportador de la industria; el entorno ambiental singular, entre otros aspectos relevantes (Pimentel, 2019, p. 48 y 49).

Finalmente se ha diseñado una ficha del producto donde se recogen las características generales que ha de tener el producto: Breve descripción, imágenes actualizadas del producto, hora de inicio y tiempo de duración, actividad a desarrollar, descripción de la actividad, y medios materiales y recursos humanos necesarios. En este sentido se ha propuesto un producto conceptual que iniciando a las 10:00am recorrerá la totalidad de los atractivos tangibles haciendo en todo momento alusión a los atractivos intangibles e 
interactuando en todo momento con la comunidad. Será un producto debidamente respaldado por medios interpretativos y deberá soportarse en el centro de visitantes planificado y diseñado afín a las características del sitio patrimonial.

\section{Conclusiones}

- El batey azucarero Jaronú es fiel exponente del patrimonio agroindustrial azucarero cubano y posee características excepcionales con altos valores patrimoniales y una imagen singular. Su ubicación cercana al destino turístico en construcción al norte de la provincia (Cayo Cruz), propicia el que sea uno de los principales recursos culturales a convertirse en un producto turístico, toda vez que es Monumento Nacional y que se han acometido labores de conservación y rehabilitación de su infraestructura que soportaría un posible producto turístico que ponga en valor a este valorado sitio patrimonial cubano.

- La definición de criterios de diseño y con ello el establecimiento de indicadores y de un programa arquitectónico para un Centro de Visitantes en el batey azucarero Jaronú, propició se diseñara un edificio con características arquitectónicas mimetizadas con la imagen industrial del entorno y con elementos figurativos singulares de la arquitectura doméstica del batey. Además, se diseñaron espacios que acogen zonas funcionales de servicio y de apoyo a las zonas de desarrollo interpretativo, todas complementadas con un recorrido temático ferroviario.

- El producto turístico conceptual “Azúcar, patrimonio y tradición en Jaronú” se desarrolló como un primer acercamiento a un producto turístico que integra y se nutre de la totalidad de los estudios precedentes sobre las características y valores patrimoniales y culturales del batey azucarero Jaronú. Se desarrolló a su vez como estudio preliminar exploratorio y fuente documental para futuros productos complementarios asociados a este sitio patrimonial.

- El presente trabajo demostró la integración multidisciplinaria de varias especialidades investigativas y académicas en torno a un objeto de investigación y mediante al apoyo en varios campos de la ciencia. En la totalidad de los ámbitos abordados se ha mantenido la premisa del vínculo cultura - turismo y la interrelación dependiente de estas con la necesaria integración de procesos para la gestión sostenible del turismo en espacios patrimoniales.

\section{Referencias bibliográficas}

Pimentel Guevara, Y. (2019). Conceptualización del producto turístico “Azúcar, patrimonio y tradición en Jaronú”. Tesis de pregrado. Licenciatura en Turismo. Universidad de Camagüey. 85p.

Brent C. B. (1984). La arquitectura de integración. España. 
Herrera Pupo, G. (2014). Concentraciones agroazucareras de Camagüey. Condicionantes de su consolidación territorial. En revista Arquitectura y Urbanismo. Vol. XXXV, No. 1, enero-abril 2014, Facultad de Arquitectura, Instituto Superior politécnico José Antonio Echeverría.

Herrera Pupo, G. (2014). Jerarquización de los recursos urbanos y arquitectónicos del patrimonio industrial azucarero de la provincia de Camagüey en Cuba. En revista electrónica Museología \& Patrimonio. Programa de Posgraduación de Museología y Patrimonio de la Universidad de Río de Janeiro. Vol. 7 Número 1.

Herrera Pupo, G. (2013). El papel de la Industria azucarera en la estructuración territorial de la provincia de Camagüey. En revista Ciência \& Trópico. Fundación Joaquim Nabuco. Vol. 36, No. 2. Recife, Brasil.

Herrera Pupo, G. (2008). El patrimonio agroindustrial azucarero de Camagüey. Tesis doctoral. CUJAE. 200p.

Herrera Pupo, G. (2006). Los bateyes azucareros de la provincia de Camagüey. En Revista Arquitectura y Urbanismo No. 2 del 2006, Vol. XXVII, pp.67-72. Ed. Facultad de Arquitectura, ISPJAE, La Habana.

Herrera Pupo, G. (2007). El patrimonio edificado de los bateyes azucareros de la provincia de Camagüey (Cuba). En Boletín de Arte. No. 28. pp. 555-570. Ed. Departamento de Historia del Arte, Universidad de Málaga, España.

Herrera Pupo, G; Pedro Bueno, Aurora. (2004). Los Asentamientos Industriales Azucareros como base de un Destino Turístico el caso de los bateyes en Camagüey, Cuba. En Creating a Framework for Tourism Policy and Destination Management. Organización Mundial del turismo. Madrid, España. Alojado en http://www.world-tourism.org/education/PDF/madrid-june2004/PedroHerrera.pdf

Barnet, M. (2005). "La cultura que generó el mundo del azúcar", Revista Cubana de Antropología Catauro, Año 6, No.11, Ed. Fundación Fernando Ortiz, La Habana.

Luís Gómez, Y. (2012). Criterios para el diseño del Centro de Visitantes del Patrimonio Agroazucarero del Batey Jaronú, en Esmeralda, Camagüey. Tesis de pregrado Licenciado en estudios socioculturales. Universidad de Camagüey. 63p.

Santisteban Moreno, A. y Hernández Marrero, Y. (2012). Centro de Visitantes para la interpretación del procesamiento agroindustrial de la caña de azúcar en Jaronú. Tesis de pregrado. Arquitectura. Universidad de Camagüey. 91p. 
Gómez, G. (2012). Metodología para el diseño de productos turísticos. Pinar del Río, Cuba: Centro de Estudios de Gerencia, Desarrollo Local y Turismo, Universidad de Pinar del Río.

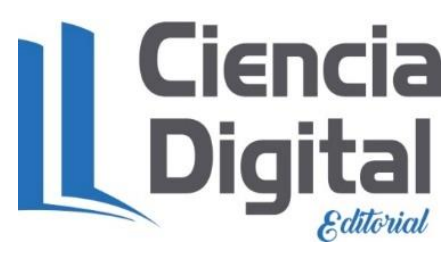




\section{PARA CITAR EL ARTÍCULO INDEXADO.}

Herrera Pupo, G., Betancourt García, M. E., \& Perera Téllez, G. (2021). Producto turístico patrimonial para el batey azucarero Jaronú, desde un enfoque multidisciplinario. Explorador Digital, 5(1), 263-280. https://doi.org/10.33262/exploradordigital.v5i1.1502

\section{LCiencia}

El artículo que se publica es de exclusiva responsabilidad de los autores y no necesariamente reflejan el pensamiento de la Revista Explorador Digital.

El artículo queda en propiedad de la revista y, por tanto, su publicación parcial y/o total en otro medio tiene que ser autorizado por el director de la Revista Explorador Digital.
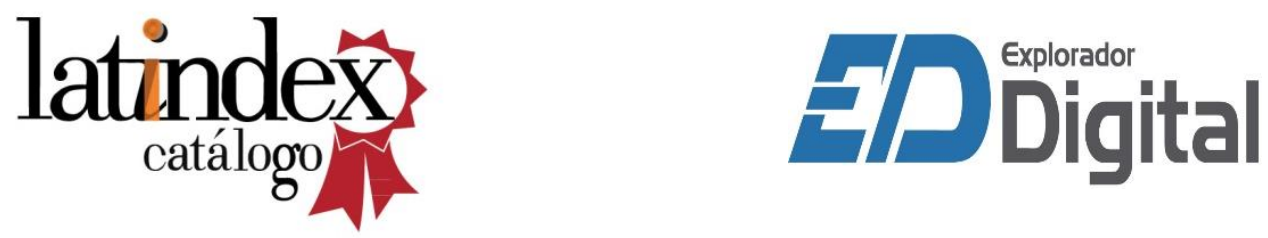